\title{
Simulating Mesoscopic Reaction-Diffusion Systems Using the Gillespie Algorithm
}

\author{
David Bernstein \\ Lawrence Berkeley National Laboratory, \\ Mail Stop 50A-1148, One Cyclotron Road, \\ Berkeley, CA 94720* \\ (Dated: December 22, 2004)
}

\begin{abstract}
We examine an application of the Gillespie algorithm to simulating spatially inhomogeneous reaction-diffusion systems in mesoscopic volumes such as cells and microchambers. The method involves discretizing the chamber into elements and modeling the diffusion of chemical species by the movement of molecules between neighboring elements. These transitions are expressed in the form of a set of reactions which are added to the chemical system. The derivation of the rates of these diffusion reactions is by comparison with a finite volume discretization of the heat equation on an unevenly spaced grid. The diffusion coefficient of each species is allowed to be inhomogeneous in space, including discontinuities. The resulting system is solved by the Gillespie algorithm using the fast direct method. We show that in an appropriate limit the method reproduces exact solutions of the heat equation for a purely diffusive system and the nonlinear reaction-rate equation describing the cubic autocatalytic reaction.
\end{abstract}

PACS numbers: 82.20.Wt, 05.40.Fb

Keywords: Gillespie algorithm, random walk, reaction-diffusion, Brownian motion

\section{INTRODUCTION}

The Gillespie algorithm [1, 2] is a stochastic method which is frequently used to simulate spatially homogeneous chemical systems with small reactant populations. Under ordinary circumstances such systems are naturally characterized by small length scales as well. Since diffusion is fast on such scales (e.g., [3]) reaction-diffusion systems are natural models of small scale, spatially inhomogeneous chemical systems. Unless diffusion coefficients are artificially decreased (as in macroscopic experiments on the formation of Turing instabilities, e.g., [4]) the range of length scale of such systems is on the order of a typical cell, roughly 0.1 to 100 microns. In systems of this size the number of reactant molecules may only be in the thousands or millions.

Recently interest has turned towards using the Gillespie algorithm to simulate such mesoscopic, spatially inhomogeneous systems $[5,6]$. The natural extension of the method is to discretize the reaction chamber into subvolumes (usually squares or cubes) and consider them to be separate chambers which are coupled by the addition of a set of reactions which model diffusion. Some form of the Gillespie algorithm is then applied to the entire system consisting of all the reactions for each subvolume plus all the diffusion events which couple them.

The purpose of this paper is to examine the numerical and computational issues involved in adding diffusion to the Gillespie algorithm in this way in more detail than has been done in the past. In particular, we show how the diffusive rate constants can be derived from a finite volume discretization of the heat equation. We then

\footnotetext{
*Electronic address: dbernstein@math.lbl.gov
}

show how the results of a stochastic simulation can be compared against exact solutions of the corresponding reaction-rate equations. This simultaneously provides a method for code testing and enables us to determine the rate of convergence of the error introduced by the spatial discretization. This puts the algorithm on a somewhat more firm computational foundation than previous works. We also investigate the regime in which the simulation contains only a single molecule and show that, again in an appropriate limit, Brownian motion is accurately simulated. The algorithm displays similar numerical properties when chemical reactions are added to the system. Although the method trivially generalizes to Cartesian meshes in any dimension, to make the analysis and numerical examples as simple and clear cut as possible we restrict our attention to systems with one space dimension. The use of the finite volume method makes likely the extension of the method to unstructured meshes in higher dimensions and various possibilities along these lines are discussed in the conclusion.

Work in this area began with the suggestion of such an application by Gillespie himself [1]. Elf et. al. [5] and Fricke and Schnakenburg [6] have implemented algorithms similar to that described in this paper but on higher dimensional Cartesian meshes. In both cases the manner in which diffusion is handled by the Gillespie algorithm differs from what is presented here. These methods are also limited to uniform meshes and systems for the which the diffusion coefficient is constant. In addition, no substantial code tests are given in either case. Lukkien et. al. [7] describe an application of what is essentially the Gillespie algorithm applied to a spatially inhomogeneous chemical system which does not strictly speaking include diffusion.

The master equation for reaction-diffusion systems was studied by Baras and Mansour [8]. They compare predictions obtained by the master equation to that obtained 
by simulation of mesoscopic systems using Bird's algorithm. In their work the domain was also one dimensional, although the boundary conditions were periodic. Their simulations were also restricted to uniform meshes and constant diffusion coefficients.

Slightly further afield but still related we find the cellular automata simulations of Weimar [9] and the pattern formation studies of Turk on triangulated surfaces [10]. Similar ideas on the relationship between continuum equations and stochastic models based on discrete nearest neighbor interactions can be found in [15-17]. The Monte Carlo part of the Gillespie algorithm appears to have been reinvented a number of times and used in a wide variety of applications, see for instance [18-20].

The underlying model assumed in what follows is that we have a set of reactant molecules immersed in a solvent and that the reactants undergo collisions with solvent molecules much more frequently than they do with each other. This is in addition to the assumption, as per the original algorithm, that non-reacting collisions between reactants greatly outnumber reacting collisions. The explicit addition of a solvent to the model is required to justify the Brownian motion of the reactants in the limit in which their populations are very small. Note that these assumptions imply that the mean free path of the reactant molecules is negligibly small, much smaller than any other length scale in the system.

The outline of this paper is as follows. Section II contains a brief review of the Gillespie algorithm while Section III shows how the diffusion part of the reactiondiffusion system is incorporated into the algorithm. Section IV gives an overview of the algorithms and data structures that make up the code. Section V details tests of the code on a purely diffusive system. In Section VI we examine a non-linear reaction-diffusion system and test the code on an exact traveling wave solution. We summarize the work in Section VII.

\section{THE GILLESPIE ALGORITHM}

The Gillespie algorithm is a well-known stochastic method in which the number of each chemical species is considered the independent variable and each reaction the system undergoes is executed explicitly. Hence the time evolution consists of a number of steps with each step being the execution of a specific reaction at a specific time. After a reaction is executed the number of molecules of each of the affected species is updated according to the reaction formula and the evolution goes on to the next step. Each reaction is assumed to be independent of the preceeding one so that the evolution of the system is a Markov process. This section contains a review of only those elements of the method which will be necessary for what follows. The reader is referred to $[1,2]$ for more detailed discussions.

The algorithm makes the following assumptions. We have a chemical system consisting of $S$ species whose state at a given time $t$ can be characterized by an integer valued tuple $\left\{S_{i}\right\}, 1 \leq i \leq S$, where $S_{i}$ is the population of species $S_{i}$. We use the convention that italicized symbols represent numbers (i.e., populations) and their nonitalicized counterparts represent names. The dynamics of the system are represented by a set of reactions of the form

$$
\alpha_{1}^{r} \mathrm{~S}_{1}+\alpha_{2}^{r} \mathrm{~S}_{2}+\ldots+\alpha_{S}^{r} \mathrm{~S}_{S} \stackrel{k_{j}}{\longrightarrow} \beta_{1}^{r} \mathrm{~S}_{1}+\beta_{2}^{r} \mathrm{~S}_{2}+\ldots+\beta_{S}^{r} \mathrm{~S}_{S}
$$

where $\alpha_{i}^{r}$ and $\beta_{i}^{r}$ are non-negative integer constants (the reactant and product coefficients respectively) and $r$ is an index which runs over the number of reactions, $1 \leq r \leq R$. Crucially, we assume that the events are exponentially distributed, i.e., the probability of reaction $j$ occurring in the time interval $t+\delta t$ to first order in $\delta t$ is $a_{j} \delta t$ where the $a_{j}$ depend only on the state of the system at time $t$. As pointed out in [11] this is only strictly valid for dimolecular reactions although it can be argued for monomolecular reactions (as will be necessary later) and holds approximately for trimolecular reactions. From these assumptions it can be shown that the reaction probability density function is given by

$$
P(\tau, j)=a_{j} \exp (-a \tau)
$$

where

$$
a=\sum_{j=1}^{R} a_{j}
$$

$P(\tau, j) d \tau$ is the probability that at time $t$ the next reaction will occur in the interval $(t+\tau, t+\tau+d \tau)$ and will be the $j$ th reaction of the system. Given the state of the system at time $t$ the core of the algorithm is a procedure for selecting the next reaction and the time it is executed in such a way that over many simulations (2) is reproduced exactly.

There exist several equivalent procedures for selecting a pair $(\tau, j)$. The merits of each of these will be discussed in Section IV. Here we give the basic algorithm, called the direct method, a version of which will be used in the final code. Let $u_{1}, u_{2} \in(0,1)$ be independent, uniformly distributed random numbers. Then the $j$ th reaction will be executed if

$$
\sum_{r=1}^{j-1} a_{r}<a u_{1} \leq \sum_{r=1}^{j} a_{r}
$$

and the time at which it is executed is $t+\tau$ where

$$
\tau=-\frac{\ln u_{2}}{a}
$$

The quantity $a_{j}$ is often referred to as the propensity for reaction $j$. Its relation to the familiar rate constant $k_{j}$ is given in $[1,2]$ but it will be convenient to summarize the results here for mono through trimolecular reactions 
(reactants : propensity)

$$
\begin{aligned}
\emptyset & : k V \\
\mathrm{~A} & : k A \\
\mathrm{~A}+\mathrm{B} & : k A B V^{-1} \\
2 \mathrm{~A} & : k A(A-1) V^{-1} \\
\mathrm{~A}+\mathrm{B}+\mathrm{C} & : k A B C V^{-2} \\
2 \mathrm{~A}+\mathrm{B} & : k A(A-1) B V^{-2} \\
3 \mathrm{~A} & : k A(A-1)(A-2) V^{-2} .
\end{aligned}
$$

Here $k$ is the appropriate rate constant and $V$ is the volume of the reaction chamber or element.

\section{DIFFUSION}

In this section we show how diffusion on an irregular grid in one dimension can be simulated by the Gillespie algorithm. As in previous work [5, 6, 8], the basic idea of the method is to divide the domain into a number of subvolumes, which we will call elements, and to consider the elements to be separate reaction chambers which are coupled together by allowing them to exchange molecules in a way designed to simulate diffusion. The coupling is formulated as an additional set of reactions which are appended to the list of chemical reactions taking place in each element. This master list is then processed by the Gillespie algorithm resulting in a coupled reactiondiffusion simulation.

In order that the original Gillespie algorithm be applicable to the chemical reactions occurring in each element we require that the concentrations there be considered uniform. This is equivalent to saying that each molecule in a given element will have an equal chance of interacting with any other molecule in the same element in a typical time interval between chemical reactions in that element. In order for this to be true the diffusion time across each element for every species should be much less than the typical reaction time. If $h$ is the length scale of an element, $D$ a typical diffusion coefficient, and $d$ the dimension of the domain, then this requirement is

$$
\tau_{D} \approx \frac{h^{2}}{2 d D} \ll \tau_{C}
$$

where $\tau_{C}$ is a typical time interval between chemical reactions. However, $\tau_{D}$ will also be the approximate diffusion time between elements, so this requirement is equivalent to saying that the typical rate of diffusion events, i.e., transfers of molecules between elements, is much greater than that of the chemical reactions inside the elements themselves.

Note that $\tau_{C}$ will be approximately the inverse of the propensity given in (6). These are of the form of a rate constant multiplied by zero or more concentration values multiplied by a single population value. Hence the propensity scales as the element population which is proportional to the element volume which in turn is propor- tional to $h^{d}$. Thus $\tau_{C}$ scales as $h^{-d}$ and so the validity of the above inequality improves as $h$ is reduced.

Whether or not (7) holds in a given simulation can easily be checked by comparing the number of diffusion events involving an element to the number of chemical reactions. In the example simulation in Section VI diffusion events typically outnumber chemical reactions by a hundred or more to one.

\section{A. The Master System}

It will be convenient to think of the reaction diffusion system not as an interaction between $S$ chemical species in a spatially inhomogeneous domain which has been subdivided into $E$ elements but as the interaction of $S E$ species in a homogeneous domain of unit volume. This larger chemical system, which we call the master system, operating in a fictitious unit volume domain enables us to use the Gillespie algorithm in its original form. The relationship between the actual chemical system and that in the master domain is the obvious one: species A is represented by $E$ species labeled $\mathrm{A}_{i}$ with the index indicating which element is occupied. The results of a simulation in the master domain can be transformed back into the problem domain when convenient, e.g., for purposes of visualization. Note that the assumption of uniform concentration in each element implies homogeneity of the master system. The use of the Gillespie algorithm on the master system differs from its ordinary usage only in the calculation of the propensity (6), in which the factor $V^{n}$ varies according to the volume of the element the reaction is taking place in.

Let $R$ be the number of reactions in the chemical system and $n$ the number of neighbors of each element. Since each element contributes $R$ chemical reactions and $S n$ diffusion reactions to the master system the total number of reactions in the master system is approximately $R^{\prime}=E(R+S n)$ (elements with faces on a boundary may contribute more or less than $S n$ reactions depending on the boundary conditions imposed there). For instance, if the chemical system contains a reaction of the form $\mathrm{A}+\mathrm{B} \rightarrow \mathrm{C}$ then the master system will contain $E$ reactions of the form $\mathrm{A}_{i}+\mathrm{B}_{i} \rightarrow \mathrm{C}_{i}$. Similarly, the ESn diffusion reactions in the master system have the form $\mathrm{A}_{i} \rightarrow \mathrm{A}_{j}$. Note that molecules in separate elements are prevented from reacting in the master system because no reactions on the master list allow them to do so.

\section{B. Diffusion in One Dimension}

Consider the case of the diffusion of a single species $U$ on a non-uniform grid in one dimension whose elements are labeled by an index $i$. The master system will then have $E$ species labeled $\mathrm{U}_{i}$. We consider a model of diffusion whereby each element exchanges molecules with its nearest neighbors only. In the master system the ex- 
change is represented by the transformation of a molecule of species $\mathrm{U}_{i}$ to one of species $\mathrm{U}_{j}$. This suggests a set of reactions for $\mathrm{U}_{i}$ of the form

$$
\begin{array}{ll}
\mathrm{U}_{i} \stackrel{k_{i, i+1}}{\longrightarrow} \mathrm{U}_{i+1}, & \mathrm{U}_{i} \stackrel{k_{i, i-1}}{\longrightarrow} \mathrm{U}_{i-1}, \\
\mathrm{U}_{i+1} \stackrel{k_{i+1, i}}{\longrightarrow} \mathrm{U}_{i}, & \mathrm{U}_{i-1} \stackrel{k_{i-1, i}}{\longrightarrow} \mathrm{U}_{i}
\end{array}
$$

where $k_{i, j}$ denotes the rate governing the reaction which transforms $\mathrm{U}_{i}$ into $\mathrm{U}_{j}$. Using the standard procedure, this set of reactions results in the reaction-rate equation for $U_{i}$

$$
\frac{d U_{i}}{d t}=-\left(k_{i, i+1}+k_{i, i-1}\right) U_{i}+k_{i+1, i} U_{i+1}+k_{i-1, i} U_{i-1}
$$

where we have used the homogeneity of the master domain.

The reader familiar with finite difference or finite element methods will recognize that the resulting set of coupled ODEs for the time evolution of the variables $U_{i}$ is in the same form as that resulting from a spatial discretization of a first order in time PDE using the method of lines, where the form of the spatial operator and the nature of the discretization are undetermined at this point. We next show that the rate constants can be set so that (9) is an approximation to the heat equation.

\section{Finite Volume Approximation}

Let $u(x, t)$ be the concentration of species $\mathrm{U}$. The two fundamental continuum equations governing diffusion are the conservation of mass

$$
\frac{\partial u}{\partial t}=-\nabla \cdot \mathbf{J}
$$

and Fick's law for the flux $\mathbf{J}$

$$
\mathbf{J}=-D(x) \nabla u .
$$

In this paper we consider the case in which $D$ is allowed to vary in space but not in time.

We start by integrating (10) over element $i$ and use the divergence theorem to evaluate the volume integral on the right hand side

$$
\frac{\partial U_{i}}{\partial t}=-\int_{i} \nabla \cdot \mathbf{J} d x=\mathbf{J}\left(c_{i}-h_{i} / 2\right)-\mathbf{J}\left(c_{i}+h_{i} / 2\right)
$$

where $c_{i}$ denotes the center of element $i$. Central to the finite volume method is the approximation used for the flux on the boundaries of each element. Here we approximate the gradient of $u$ at the left boundary by

$$
\begin{aligned}
\nabla u\left(c_{i}-h_{i} / 2\right) & \approx \frac{u\left(c_{i}\right)-u\left(c_{i-1}\right)}{c_{i}-c_{i-1}} \\
& =\frac{1}{c_{i}-c_{i-1}}\left(\frac{U_{i}}{h_{i}}-\frac{U_{i-1}}{h_{i-1}}\right) .
\end{aligned}
$$

Performing the same procedure at the right boundary, employing (11), and inserting into (12) yields

$$
\begin{aligned}
\frac{\partial U_{i}}{\partial t}= & -U_{i}\left(\frac{D_{i, i-1}}{h_{i}\left|c_{i}-c_{i-1}\right|}+\frac{D_{i, i+1}}{h_{i}\left|c_{i}-c_{i+1}\right|}\right) \\
& +U_{i-1} \frac{D_{i-1, i}}{h_{i-1}\left|c_{i}-c_{i-1}\right|}+U_{i+1} \frac{D_{i+1, i}}{h_{i+1}\left|c_{i}-c_{i+1}\right|}
\end{aligned}
$$

where $D_{i, j}$ is the diffusion coefficient evaluated at the interface between elements $i$ and $j$. This suggests setting the rate constants in (9) to

$$
k_{i, j}=\left\{\begin{array}{cl}
\frac{D_{i, j}}{h_{i}\left|c_{i}-c_{j}\right|}, & |i-j|=1 \\
0, & \text { otherwise }
\end{array} .\right.
$$

The diffusion coefficient at the faces of the mesh is evaluated using the well-known weighted harmonic average (e.g., $[26,27])$

$$
D_{i, j}=\left(\frac{1}{\left|c_{i}-c_{j}\right|} \int_{c_{i}}^{c_{j}} \frac{d s}{D(s)}\right)^{-1}
$$

where the integral is along the straight line joining the centers of the two elements. For $D(x)$ piecewise constant in one dimension this is

$$
D_{i, i+1}=\left[\frac{1}{h_{i}+h_{i+1}}\left(\frac{h_{i}}{D_{i}}+\frac{h_{i+1}}{D_{i+1}}\right)\right]^{-1}
$$

which in the case of a uniform grid reduces to the usual harmonic average $D_{i, i+1}=\left(1 / D_{i}+1 / D_{i+1}\right)^{-1}$.

If we consider $u_{i}=U_{i} / h_{i}$ to be element-centered variables and write (14) in terms of $u_{i}$ then the truncation error term on the right hand side is $O(h)$ where $h$ indicates the size of the largest element in the mesh. However we will see later that the $L_{\infty}$ norm of the error of the overall scheme converges as $h^{2}$. This increase in the rate of convergence over that of the truncation error is known as supraconvergence and is a common feature of finite volume approximations to systems based on conservation laws [12].

Thus a solution to the system (9) will generate a set of element centered concentration values $u_{i}$ having an error compared to the exact solution of the heat equation which will decrease as $h^{2}$ assuming the time integration is exact. The Gillespie algorithm is such an exact time integrator in the sense that the only error associated with it is a sampling error.

Note that on a uniform grid with a constant diffusion coefficient (15) reduces to $k_{i, j}=D h^{-2}$ which is the expression used in $[5,6]$. In addition, (9) reduces to

$$
\frac{d U_{i}}{d t}=D \frac{U_{i+1}-2 U_{i}+U_{i-1}}{h^{2}}
$$

which is the familiar second order centered rule for finite differences. 


\section{Boundary Conditions}

The natural condition for a closed microchamber is that no molecules move through the boundary. Since the rate constants in (15) are associated with faces of the mesh this condition is enforced by setting the rate constants associated with faces on the boundary to zero. That this condition yields an approximation to a Neumann condition can be seen by rewriting the flux approximation (13) in terms of the rate constants and noticing that the flux across the boundaries is zero.

Dirichlet conditions can be modeled as follows. Let the leftmost interval have index 1 and let a fictitious element to its left have index 0 . The rate equation for $\mathrm{U}_{1}$ is

$$
\frac{d U_{1}}{d t}=-\left(k_{1,2}+k_{1,0}\right) U_{1}+k_{2,1} U_{2}+k_{0,1} U_{0} .
$$

The second and fourth terms represent molecules leaving and entering the domain through the left boundary which can be represented by the reactions

$$
\mathrm{U}_{1} \stackrel{k_{1,0}}{\longrightarrow} \emptyset, \quad \emptyset \stackrel{k_{0,1}}{\longrightarrow} \mathrm{U}_{1} .
$$

The two rate constants can be set by choosing a reasonable value for $h_{0}$, one which is comparable to other values in the system. For instance, choosing $h_{0}=h_{1}$ gives

$$
k_{1,0}=\frac{D_{1,0}}{h_{1}^{2}}, \quad k_{0,1}=D_{0,1} \frac{U_{0}}{h_{0} h_{1}}=\frac{D_{0,1} u_{0}}{h_{1}}
$$

where $u_{0}$ is the boundary value of the concentration. The choice of $h_{0}$, and consequently $k_{1,0}$ and $k_{0,1}$, effectively determines how rapidly the Dirichlet condition is able to respond to changes in the concentration near the boundary.

\section{ALGORITHMS AND DATA STRUCTURES}

The number of reactions in the master system can easily be in the millions for even a modest mesh and a small set of chemical reactions. Thus efficient data storage and fast algorithms are required to make the simulation feasible. Fortunately this work has already been done in order to address the needs of simulating large chemical systems with many reactions. In this section we give a brief overview of these methods and their implementation in the code which is used in the rest of the paper.

\section{A. The Fast Direct Method}

As is well-known both of Gillespie's original algorithms for computing the next reaction and its execution time, called the direct method and the next reaction method, are not efficient when the number of reactions is very large. As a result a series of fast versions of both algorithms have been developed. The fast version of the direct method involves a binary tree while the fast version of the next reaction method involves a priority queue. Both methods require $\log R^{\prime}$ time to produce a pair $(\tau, j)$. They differ primarily in two respects: i) the constant in front of the $\log R^{\prime}$ term, and ii) the number of uniform random numbers needed.

In our experience, (ii) is not a concern since the speed of the random number generator is not a bottleneck in the simulation. In the examples below we have used the 32-bit numerical recipes routines RAN2 and RAN3 [22] and the 64-bit routine lsfr258 [23]. The latter is roughly three times slower than RAN3 but the resulting simulation time is only a few percent longer. Most of the simulation time is spent recomputing the rates of affected reactions and updating the corresponding data structures.

We have chosen to use the fast direct method over the fast next reaction method of Gibson and Bruck [14] even though it is likely to be inferior regarding point (i). The reason for this is that we are looking ahead to the addition of automatic mesh refinement. AMR will involve the dynamical addition and subtraction of reactions from the system and it is unclear how to maintain the priority queue under these conditions. The maintenance of the binary tree in the direct method, however, is conceptually straightforward since reactions appear as leaves of the tree, which can easily be added and removed.

Since details of the binary tree used in the method are given in $[13,14]$ we will not repeat them here but will merely make the following observations. Because of the nearest neighbor model of diffusion the dependency graph of the master system remains sparse so that updating all of the reactions dependent on the outcome of a given reaction is a constant time operation. We also note that the current implementation in the code described below is that of a straightforward binary tree. This means that the depth of the tree can be as high as 20 or 25 , i.e., the tree may have tens of millions of leaves. At this depth, these types of trees often suffer in performance due to cache related problems. Although we have not observed this in the examples described below it seems prudent to warn the reader that they may occur in higher dimensional simulations with larger meshes and more complex chemical systems.

\section{B. The Code}

The code used in the examples below is written in ANSI $\mathrm{C}++$ and relies heavily on the standard template library. The code was built using using CodeWarrior V9, optimized for speed, and run on a single processor of a dual $2 \mathrm{GHz}, 1.5 \mathrm{~Gb}$ RAM, macintosh G5 running OS 10.3.5. In a typical simulation with a moderately large tree $\left(10^{5}\right.$ leaves $)$ the code executes about $3 \times 10^{5}$ reactions per second. 


\section{Complexity}

The running time of the code is a function of $E, R$, $S$, and $n$, as well as the total number of molecules in the simulation. However, as stated above the model assumes that the slowest diffusion process in a given element is much faster than the fastest chemical process. This means that in any given simulation the number of diffusion events will greatly outnumber the number of chemical reactions. Hence the running time of the code will be determined primarily by the diffusion part of the simulation and this in turn will be determined by the species which undergoes the most diffusion events. Thus the complexity of the algorithm will depend primarily on two quantities: the number of molecules of this species and the number of elements in the mesh. In the case where all species have similar diffusion coefficients this number is just the total number of all species. Hence in this section we will examine the scaling of the running time of the code in the case of the pure diffusion of a single species. Since the formulae here are easily generalized to more than one space dimension we do so; the reader should keep in mind that the examples in the sections which follow are all performed with $d=1$.

Consider a purely diffusive system involving $N$ molecules of one species A. From (5) we see that a typical time step has size

$$
\tau \approx\left(\sum_{i=r}^{R^{\prime}} a_{r}\right)^{-1}
$$

If $h$ is a typical element length scale then according to (6) and (15) $a_{r} \propto A_{i} h^{-2}$ where $A_{i}$ is the number of molecules in element $i$. There are $n$ diffusion reactions per element, with $n$ the number of neighbors of each element, so that $R \approx n E$ which gives

$$
\tau \propto\left(\sum_{i=1}^{E} A_{i} h^{-2}\right)^{-1} \propto \frac{h^{2}}{N} .
$$

For a uniformly refined mesh the number of elements scales as $h^{-d}$, hence a run to time $t$ has number of steps

$$
N_{\mathrm{S}} \approx \frac{t}{\tau} \propto \frac{N}{h^{2}} \propto N E^{2 / d}
$$

and since each step requires time proportional to $\log E$ the run time $T_{\mathrm{r}}$ scales as

$$
T_{\mathrm{r}}=O\left(N E^{2 / d} \log E\right) .
$$

The scaling of $N_{\mathrm{S}}$ and $T_{\mathrm{r}}$ is shown in Figure 1 for a series of runs on the unit interval. There is one species with $N=1000$ and $D=1$. The initial distribution is uniform and the code is run to $T=0.1$. The run time is in seconds and and the number of steps is in units of $10^{5}$. The data is averaged over five runs.
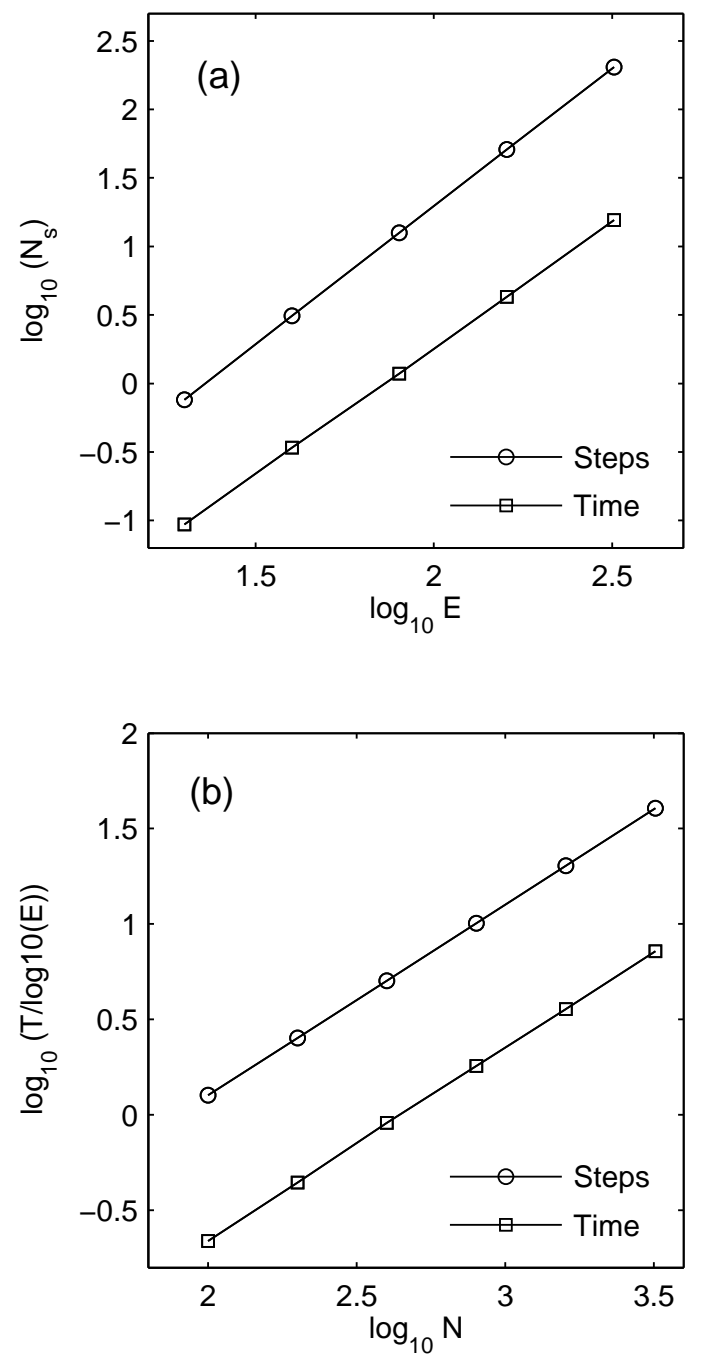

FIG. 1: Illustration of relations (24) and (25) in one dimension. Figure (a) shows $\log N_{\mathrm{S}}$ and $\log \left(T_{\mathrm{r}} / \log E\right)$ v.s. $\log E$ v.s. $\log E$ for $N=1000$. Figure (b) shows the same quantities v.s. $\log N$ for $E=80$. Least squares fits to the data in (a) give slopes of 2.02 and 1.84 for $N_{\mathrm{S}}$ and $T_{\mathrm{r}} / \log E$ respectively while fits to both curves in (b) give unit slope.

It is instructive to compare (25) with the complexity of a simple finite element or finite difference code for approximating solutions of the heat equation. For the purposes of this comparison we ignore the important issues of stiffness, automatic time stepping, mesh refinement, etc. We assume the PDE method uses an implicit scheme with update time per step of $O(E \log E)$. In order that the comparison be meaningful we require the $\mathrm{PDE}$ method to be second order in space and time, i.e., that is has a truncation error which scales as $O\left(h^{2},(\Delta t)^{2}\right)$. Hence in order that this error scale as $h^{2}$ we should have $\Delta t \propto h$ which leads to $N_{\mathrm{S}} \propto h^{-1} \propto E^{1 / d}$ so that the run 
time should scale as

$$
T_{\text {pde }}=O\left(E^{1+1 / d} \log E\right) .
$$

The ratio of this time to (26) is

$$
\frac{T_{\mathrm{r}}}{T_{\mathrm{pde}}}=O\left(N E^{-1+1 / d}\right)
$$

so that for $N$ small the stochastic method is favored in all dimensions $d>1$. On the other hand, for $E$ fixed the scaling favors the PDE method in the limit of large $N$, which is precisely the limit in which the continuum approximation becomes valid. This is not surprising since the magnitude of a concentration appears as an amplitude in a system of PDEs so it does not affect the complexity. Note that here we are comparing the simulation times for a single run of both methods. In some cases, as in the sections which follow, it will be useful to compute an average over a very large number of runs. In this case the average solution may be meaningfully compared to the result of the continuum approximation method and it would be appropriate to append a factor of $M^{2}$ to $T_{\mathrm{r}}$. In this case the stochastic method suffers considerably because of the slow convergence of the sampling error.

\section{TESTING DIFFUSION}

The purpose of this section is to determine whether or not the simulation of diffusion by the Gillespie algorithm in the manner outlined above works. We do this by performing two tests: (i) comparison of the simulation to an exact solution of the heat equation and (ii) comparison of the simulation to the statistics of Brownian motion. While these tests are essentially straightforward, there are one or two fine points that need to be mentioned before we begin.

First, note that the result of a single simulation is a randomly generated piecewise integer function over the grid. Hence in order that a meaningful comparison be made to a smoothly varying exact solution the results must be averaged over many simulations. It is important to understand that this average solution is not guaranteed to be in some sense representative of the final state of the system. This will happen for instance in the case where the system undergoes a bifurcation due to an instability (as in pattern formation via a Turing instability). In general, we should anticipate that this situation will arise whenever we have a system which is sensitive to initial conditions. However, there are other systems for which the average solution does converge in a sensible way and this makes them ideal candidates for code testing.

For these cases, averaging over simulations makes sense and for the type of test described below the fluctations about the average solution can be regarded as a type of sampling error which can be decreased by increasing the number of simulations. In this case the only property of the fluctuations we will be interested in is their mean. In general though other statistical properties of the fluctuations will be more important. Indeed, one of the attractive properties of the Gillespie algorithm is the possibility that it can reproduce in a natural way the statistical properties of real systems [11].

\section{A. Initial Data}

To begin a simulation the code reads a data file containing among other things a set of user specified concentration functions which determine the average initial conditions. For a single species A let this function be $a(x)$. The initial data of a simulation is the initial distribution of molecules among the elements, which, as noted above, is a piecewise integer function over the domain. This function cannot be identical for each simulation because on average it would not represent $a(x)$ very well. In this section we show how the initial data is generated from a probability distribution so that when averaged over many instances it does converge to $a(x)$.

Let $A_{i, \mu}$ be the number of molecules initially placed in element $i$ for simulation $\mu$. The procedure for generating $A_{i, \mu}$ is as follows. First the total number of molecules in the simulation is computed from $a(x)$. In some cases the total number of molecules can be computed exactly but in general an approximation

$$
\bar{A}=\int a(x) d x=\sum_{i=1}^{E} \int_{i} a(x) d x \approx \sum_{i=1}^{E} \bar{A}_{i}
$$

can be used, where $\bar{A}_{i}$ is a suitable quadrature of $a(x)$ over element $i$. Even though an exact expression is available for the test systems below, in what follows the trapezoidal rule is used to compute $\bar{A}_{i}$ in order that the results be more representative of what might be encountered in practice. $\bar{A}$ is of course non-integer in general. Let the total number of molecules for simulation $\mu$ be generated randomly from $\bar{A}$ by

$$
A_{\mu}=\lfloor\bar{A}\rfloor+ \begin{cases}1, & u<\operatorname{frac}(\bar{A}) \\ 0, & u \geq \operatorname{frac}(\bar{A})\end{cases}
$$

where $u$ is a uniform random number and $\lfloor\bar{A}\rfloor$ and frac $(\bar{A})$ are the integer and fractional parts of $\bar{A}$ respectively. The function $P_{i}=\bar{A}_{i} / \bar{A}$ is treated as a discrete probability distribution which is sampled $A_{\mu}$ times, the outcome of each sample being used to increment $A_{i, \mu}$ (hence $A_{\mu}=\sum_{i} A_{i, \mu}$ ).

It is clear that this method of producing initial data will converge to the continuous function $a(x)$ in the limit $M \rightarrow \infty$ in the sense that the averages

$$
A_{M}=\frac{1}{M} \sum_{\mu=1}^{M} A_{\mu}, \quad A_{i, M}=\frac{1}{M} \sum_{\mu=1}^{M} A_{i, \mu}
$$




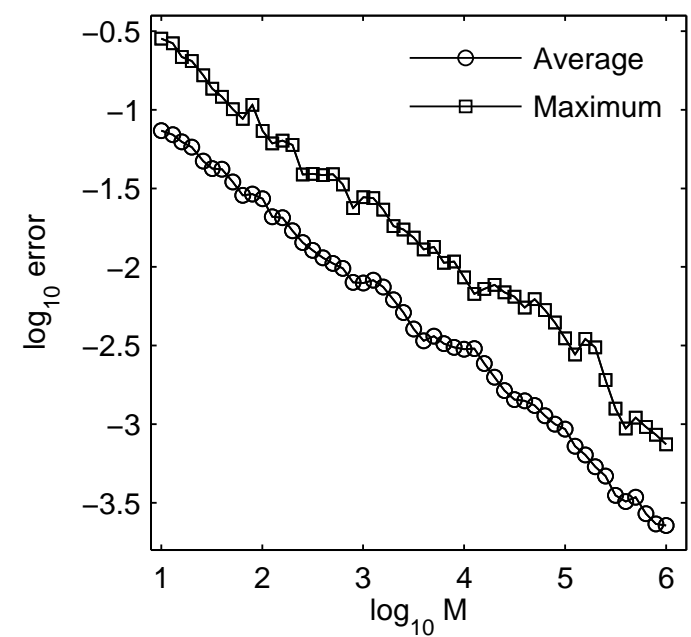

FIG. 2: Convergence of the error measures (33) for initial data on the unit interval. In this example $E=50, n=3$, and $\tilde{A}=500$. Least squares fits to the data give a slope of -0.5 and -0.48 for the average and maximum error respectively.

will obey

$$
\left|A_{M}-\bar{A}\right| \propto M^{-1 / 2}, \quad\left|A_{i, M}-\bar{A}_{i}\right| \propto M^{-1 / 2} .
$$

To see this in practice consider the error in $A_{i, \mu}$ and denote by $e_{i, M}$ its average over $M$ runs

$$
e_{i, M}=\frac{1}{M} \sum_{\mu=1}^{M}\left(1-\frac{A_{i, \mu}}{\bar{A}_{i}}\right) .
$$

We look at the average and maximum of $e_{i, M}$ over the mesh

$$
\bar{e}_{M}=\frac{1}{E} \sum_{i=1}^{E}\left|e_{i, M}\right|, \quad \hat{e}_{M}=\max _{1 \leq i \leq E}\left|e_{i, M}\right| .
$$

Figure 2 shows the convergence of these two quantities for initial data given by (35) with $n=3$ and $\tilde{A}=500$. The mesh consists of 50 evenly spaced elements.

\section{B. Time Evolution with Constant Diffusion Coefficient}

In this case the concentration $a(x, t)$ obeys

$$
\frac{\partial a}{\partial t}=D \frac{\partial^{2} a}{\partial x^{2}}, \quad \frac{\partial a}{\partial x}(0)=\frac{\partial a}{\partial x}(1)=0 .
$$

Setting $D=1$, an exact solution on the unit interval $[0,1]$ satisfying the boundary conditions is

$$
a(x, t)=\tilde{A}\left(1+1 / 2 e^{-\pi^{2} n^{2} t} \cos (n \pi x)\right)
$$

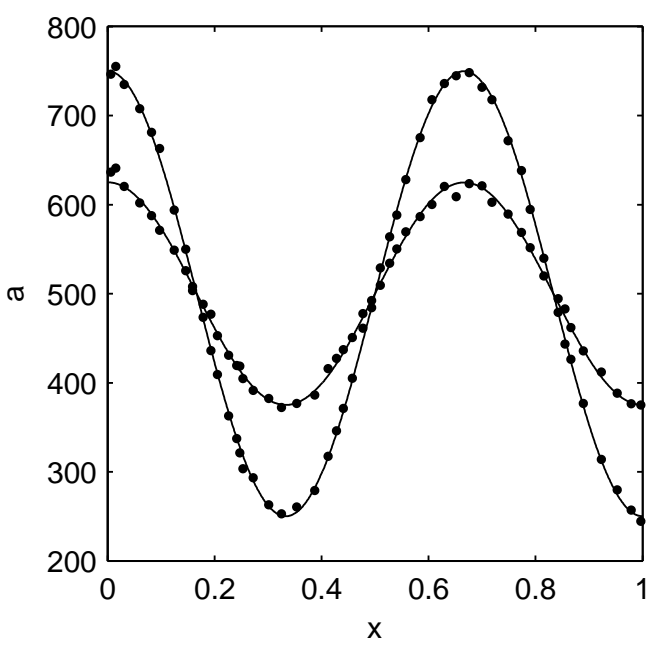

FIG. 3: Average solution of the initial data (top curve) and evolution to $T=0.0078$ (middle curve) on a randomly spaced grid. Here $n=3, E=50, D=1, \tilde{A}=500$, and $M=1000$. The solid circles are the average concentration while the solid line is the exact solution (35).

where $n$ is a non-negative integer. Note that the total number of $\mathrm{A}$ is $\tilde{A}$.

In this section we look at the convergence of the error measures (33) for the time evolution. To do this we replace $\bar{A}_{i}$ in $(32)$ with $\bar{A}_{i}(t)$ computed from (35). From the exact solution we see that the difference between the maximum and minimum value of $a(x, t)$ will decrease from $\tilde{A}$ to $\tilde{A} / 2$ in time

$$
T=\frac{\ln 2}{n^{2} \pi^{2}}
$$

which gives us a reasonable time at which to end the simulation. Recall that each simulation is begun with a randomly generated set of initial data using the algorithm in the previous section. We can define an average concentration over $M$ runs as

$$
a_{i, M}=\frac{1}{M} \sum_{\mu=1}^{M} A_{i, \mu} h_{i}^{-1} .
$$

A typical plot of $a_{i, M}$ for 1000 simulations is shown in Figure 3. In this figure $n=3, \tilde{A}=500$, and the mesh consists of 50 randomly sized elements created by randomly generating $0.1 \leq h_{i} \leq 1$ and scaling to the unit interval.

Next we inspect the error measures (33). We set $n=1$ which gives $T \approx 0.07$. Figure 4 shows the two error measures for a set of $10^{4}$ runs to $T=0.07$ on a mesh with 50 equal size elements and with 50 randomly sized elements. The number of molecules is set at $\tilde{A}=500$ in both cases. 

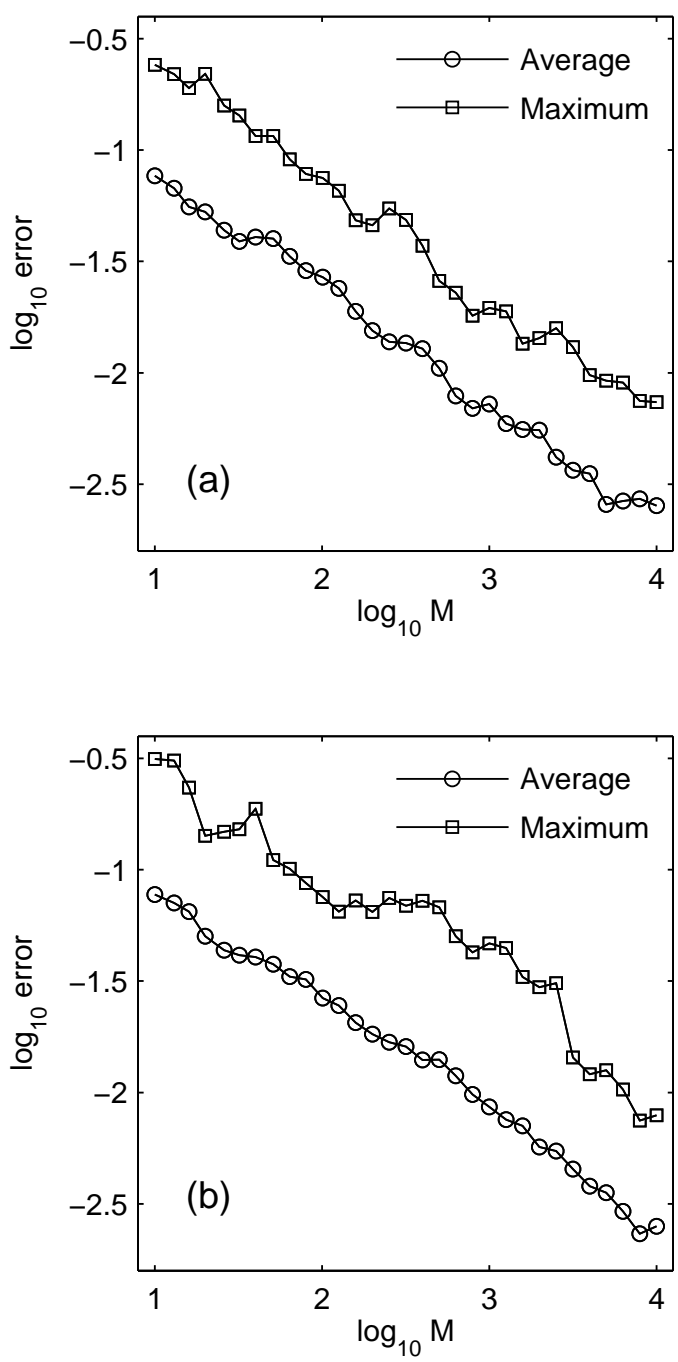

FIG. 4: Convergence to the exact solution of the heat equation on an evenly spaced (a) and randomly spaced (b) grid. Fits to $\bar{e}_{M}$ yield slopes of -0.52 and -0.5 respectively. The diffusion coefficient is set to unity in both cases.

Again the slope is about $-1 / 2$ in both cases. As in Figure 2, it appears that the curves in Figure 4 are converging to zero error in the limit $M \rightarrow \infty$. However, unlike the results for the initial data, we show in Figure 5 that this is not the case. Here the time evolution is performed on a coarser grid and we use $n=3$ in (35). In Figure $5(\mathrm{a})$ we see that $\bar{e}_{M}$ for $E=20$ equal size elements levels off at around 0.002 at $M \approx 10^{4}$ and does not decrease further. Figure 5(b) shows that this asymptotic sampling error is related to the mesh spacing $h$ and scales as $h^{2}$.
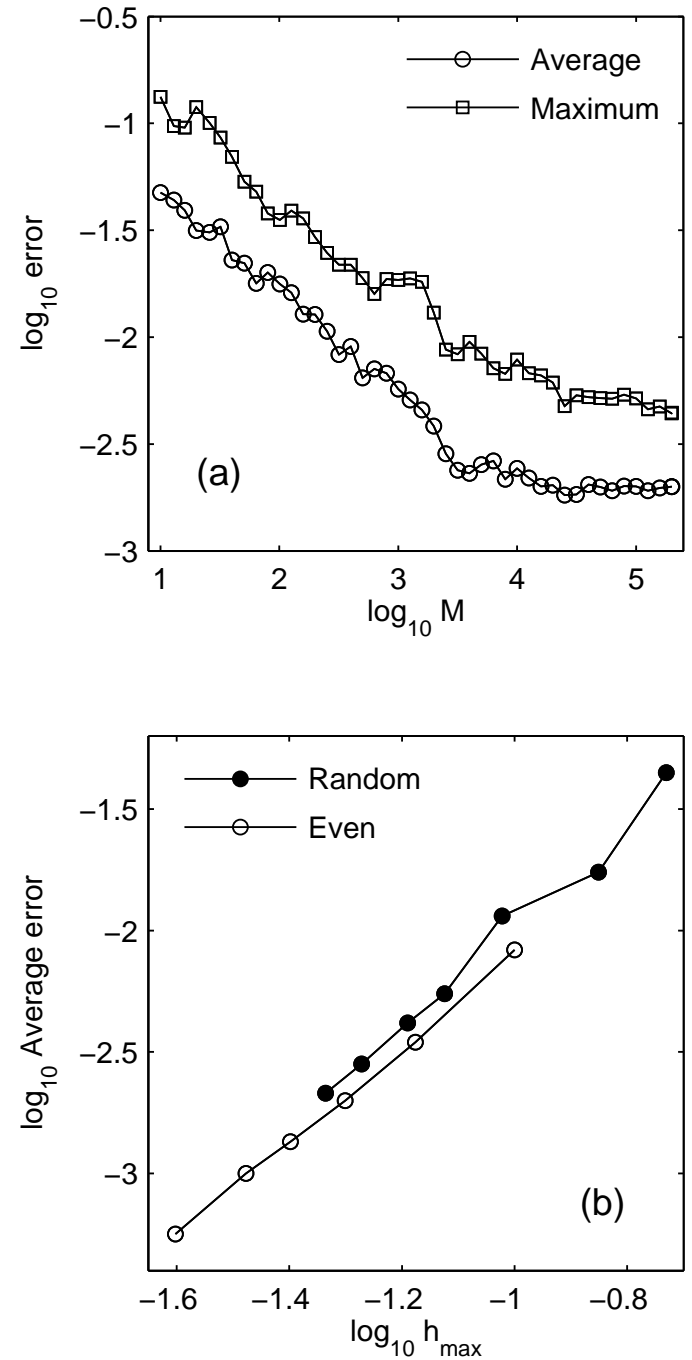

FIG. 5: Demonstration of the spatial discretization error. In these examples $n=3$ and $T=\ln 2 /\left(9 \pi^{2}\right) \approx 0.0078$. In (a) we show the error measures for $E=20$ on an evenly spaced grid. The asymptotic value of $\bar{e}_{M}$ is about 0.002 . In (b) we plot the asymptotic value of $\bar{e}_{M}$ v.s. the maximum element size $h$ for a series of evenly and randomly spaced grids with $10 \leq E \leq 40$. Fits to the data gives slopes of 1.92 and 2.11 respectively.

\section{Time Evolution with Discontinuous Diffusion Coefficient}

In this section we repeat the tests on the unit interval above but with the inhomogeneous diffusion coefficient

$$
D(x)= \begin{cases}1, & 0 \leq x \leq 0.5 \\ 5, & 0.5<x \leq 1\end{cases}
$$

$D(x)$ is chosen to be discontinuous rather than smoothly varying because we anticipate that this case will be the one most likely encountered in practice. 


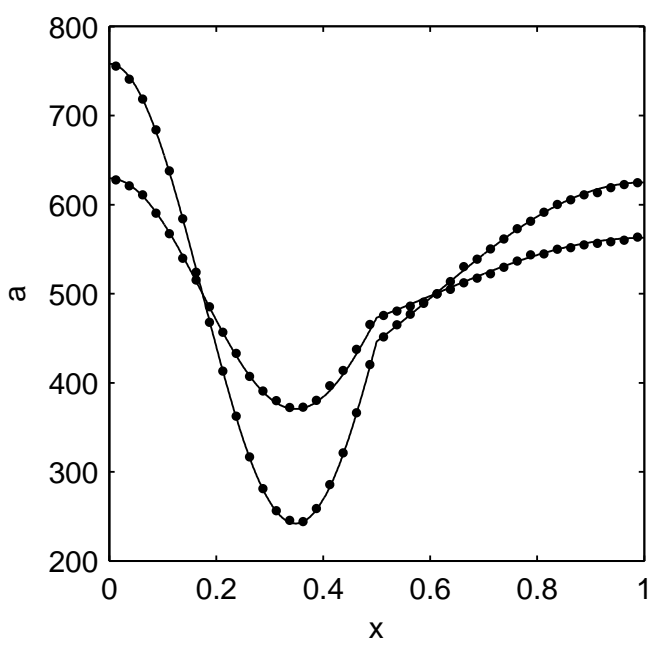

FIG. 6: The average solution (solid circles) is shown against the exact solution (solid line) for a series of runs with the discontinuous diffusion coefficientf (38). The top curve is the initial data while the middle curve is the data at $T=0.0085$. In this case $E=40, M=10^{4}, \tilde{A}=500$ and the mesh is evenly spaced.

The eigenfunctions and eigenvalues of the weak form of

$$
\frac{\partial}{\partial x}\left(D(x) \frac{\partial f}{\partial x}\right)-\lambda^{2} f=0
$$

with Neumann boundary conditions are

$$
f_{n}(x)= \begin{cases}\rho_{n} \cos \left(\lambda_{n} x\right), & 0 \leq x \leq 0.5 \\ \cos \left(\lambda_{n}(1-x) / \sqrt{5}\right), & 0.5<x \leq 1\end{cases}
$$

with $\lambda_{n}$ given by the roots of

$$
(1 / \sqrt{5}) \tan (\lambda / 2)+\tan (\lambda / 2 \sqrt{5})=0
$$

and $\rho_{n}$ given by

$$
\rho_{n}=\frac{\cos \left(\lambda_{n} / 2 \sqrt{5}\right)}{\cos \left(\lambda_{n} / 2\right)} .
$$

Similar to the above, we choose the following linear combination of the zeroth and second eigenfunctions

$$
a(x, t)=\tilde{A}\left(1+1 / 4 e^{-\lambda_{2}^{2} t} f_{2}(x)\right),
$$

where $\lambda_{2} \approx 9.0065$ and $\rho_{2} \approx 2.0651$, and set the stopping time to $T=\ln 2 / \lambda_{2}^{2} \approx 0.0085$.

Figure 6 shows the average solution over $10^{4}$ runs against the exact solution (43) on an evenly spaced 40 element mesh. Measuring the spatial error with the method outlined above shows that the convergence is $O\left(h^{2}\right)$ in this case as well.

\section{Stopping Time}

Each simulation in the above experiments is stopped when the cumulative time exceeds the set time $T$. This means that the actual stopping time, and the time at which the comparison with (35) is made, exceeds (36) by a small random amount. Hence the error measures $\bar{e}_{M}$ and $\hat{e}_{M}$ are not computed with respect to a fixed value of $T$. In this section we show that the variation in the stopping time is small compared to $T$, which provides an a posteriori justification of this procedure.

We expect that if the stop time is $T+\Delta T$ then according to $(24)$

$$
\Delta T \propto \frac{1}{N E^{2 / d}}
$$

A typical spread of $\Delta T$ for a series of 1000 simulations similar to that in the previous section is shown in Figure $7(\mathrm{a})$. Since $\Delta T$ should be exponentially distributed we expect that if $w$ is the width of an exponential fitted to the data then we should have $\Delta T \approx w \propto N^{-1}$. This is shown in the Figure $7(\mathrm{~b})$. Even on a small grid with a modest number of molecules the average stopping time exceeds $T$ by only a few parts in $10^{5}$.

\section{E. Random Walks}

The relationship between diffusion, brownian motion, and random walks is well known. In this section we look at the motion of a single molecule propagating on a mesh by the algorithm above. If the molecule undergoes a random walk starting at the origin at time $t=0$ the distribution of its position at time $t$ is given by

$$
P(\mathbf{r}, t)=\frac{1}{(4 \pi D t)^{d / 2}} \exp \left(-\frac{r^{2}}{4 D t}\right)
$$

which has the well-known expectation value

$$
\left\langle r^{2}\right\rangle=2 d D t
$$

Let the molecule begin the simulation at $t=0$ in an element with center $c_{0}$ and end the simulation at time $t$ in an element with center $c_{t}$. A measure of (46) over $M$ runs is

$$
m_{M}=\frac{1}{M} \sum_{\mu=1}^{M} \frac{\left|c_{t}-c_{0}\right|_{\mu}^{2}}{2 d D t}
$$

$m_{M}$ only measures the mean value of the distribution. To see the whole distribution note that in one dimension the probability of the molecule ending up in an interval of width $\Delta$ centered at $x$ is

$$
p(x, t, \Delta)=\left.\operatorname{erf}\left(\frac{x}{\sqrt{4 D t}}\right)\right|_{x-\Delta / 2} ^{x+\Delta / 2} .
$$



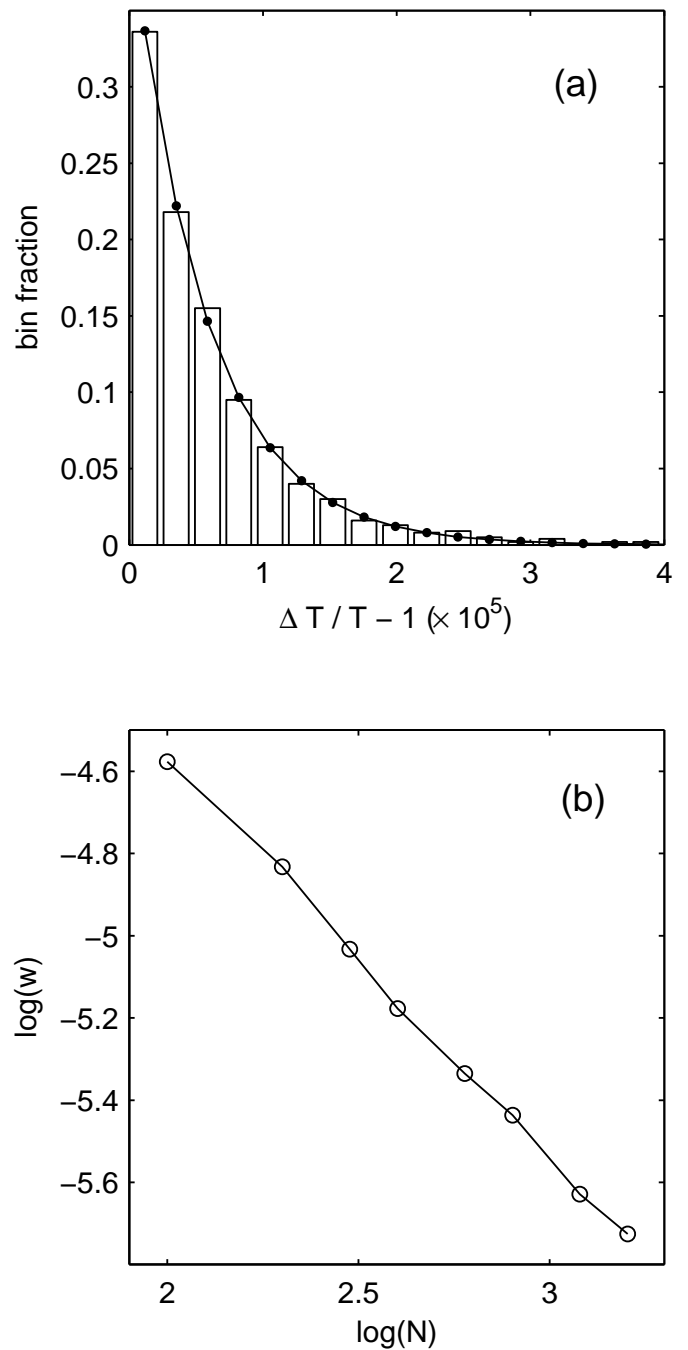

FIG. 7: The distribution of stopping times from a series of 1000 runs is shown in Figure (a). An exponential fitted to the data is shown by the solid circles. The initial data is (35) with $n=1, N=500$, and the stopping time is set to $T=0.07$. The mesh consists of 50 evenly spaced elements. In (b) we show the width $w$ (the $1 / e$ point) of an exponential distribution fitted to the data as a function of $N$. The fit was done to the first 25 of 50 bins for each value of $N$.

With $D=1$ the typical time to diffuse from the center of the unit interval to the boundary is $T_{b}=1 / 8$. We set the stopping time to a small fraction of this, $T=0.005$, in order to make it very unlikely that the molecule reach one of the boundaries by the end of the run. The results for (47) and (48) of the random walk of a single molecule in one dimension for a series of $5 \times 10^{4}$ runs are shown in Figure 8. The spatial discretization error discussed above should be kept in mind when considering this figure. We expect the error in the random walk to have the same $O\left(h^{2}\right)$ dependency as the previous results.
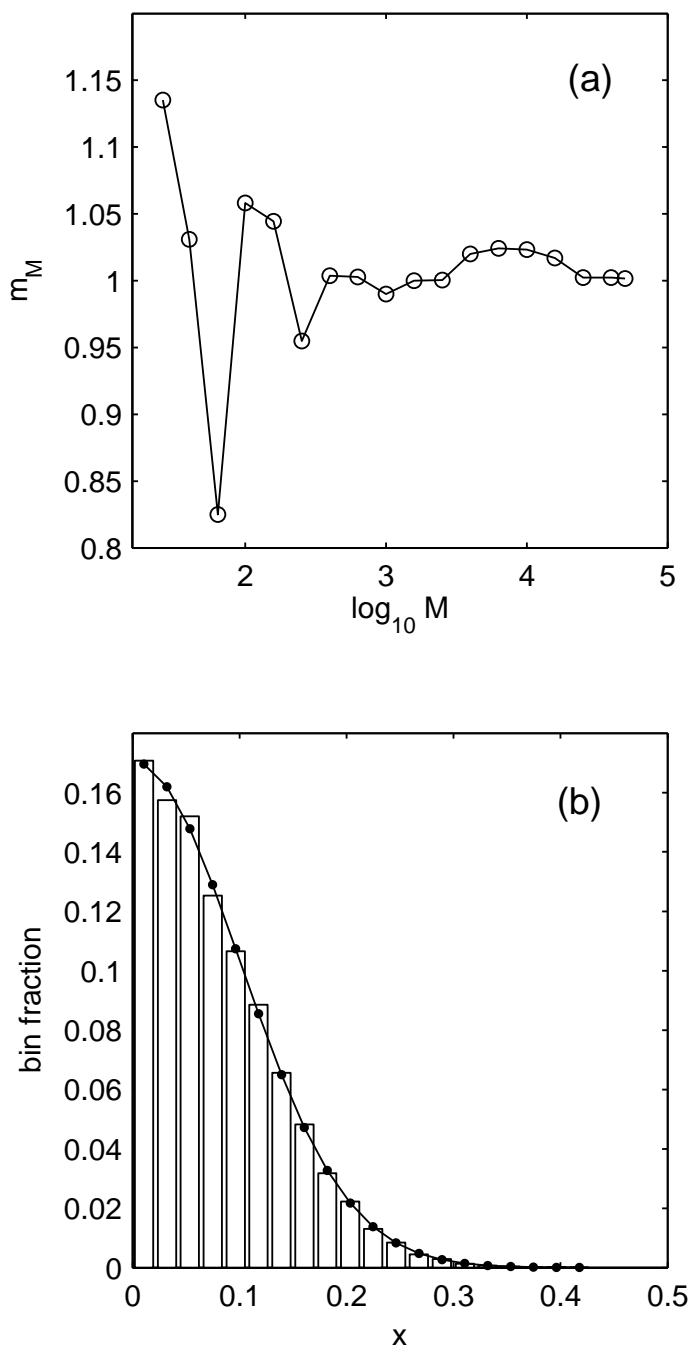

FIG. 8: Figure (a) shows $m_{M}$ for a series of $5 \times 10^{4}$ runs. The mesh consists of 1000 equally sized elements, $D=1$, and the run time is $T=0.005$. Figure (b) shows the distribution of $x=\left|c_{t}-c_{0}\right|$ at $T=0.005$. The solid circles are the exact distribution (48).

\section{NON-LINEAR WAVE PROPAGATION}

The cubic autocatalytic reaction (see, e.g., [21])

$$
\mathrm{A}+2 \mathrm{~B} \stackrel{k}{\rightarrow} 3 \mathrm{~B}
$$

is at the heart of several interesting and well-studied nonlinear chemical systems, the archetypal example being the Brusselator $[24,25]$. In one dimension it becomes a good candidate for algorithm testing as a reactiondiffusion system because of the existence of a traveling wave solution whose wavefront has a constant velocity and shape. In addition we will see that the averaging procedure outlined in the previous section appears to make 
sense for this system with the initial conditions described below.

\section{A. Wave Propagation}

Let the two species A and B have the same diffusion coefficient $D$ and concentrations $a(x, t)$ and $b(x, t)$. If the initial value of $a+b$ is constant in space then $a+b$ is will be constant in time as well and the reaction-rate equation governing $b$ reduces to

$$
\frac{\partial b}{\partial t}=D \frac{\partial^{2} b}{\partial r^{2}}+k b^{2}\left(a_{0}-b\right)
$$

where $a_{0}$ is the initial value of $a+b$. This can be put in dimensionless form by the scaling

$$
\beta=\frac{b}{a_{0}}, \quad \tau=k a_{0}^{2} t, \quad x=r \sqrt{\frac{k a_{0}^{2}}{D}} .
$$

An exact traveling wave solution for $\beta$ of (50) is

$$
\beta(z)=\frac{\exp \left[-(1 / \sqrt{2})\left(z-z_{0}\right)\right]}{1+\exp \left[-(1 / \sqrt{2})\left(z-z_{0}\right)\right]}
$$

where

$$
z=x-c \tau, \quad c=\frac{1}{\sqrt{2}}
$$

and $z_{0}$ is a constant of integration. The boundary conditions satisfied by (52) are

$$
\lim _{z \rightarrow+\infty} \beta(z)=0, \quad \lim _{z \rightarrow-\infty} \beta(z)=1 .
$$

The location of the wavefront is given by $\beta=1 / 2$ or $x-c \tau-z_{0}=0$, which at $\tau=0$ implies $x=z_{0}$.

Our aim in this section is to compare the output of the code to the exact solution (52). However, as discussed in Section III D the simulation requires boundary conditions and (52) on a finite domain $\left[0, x_{b}\right]$ does not satisfy either a Dirichlet or Neumann condition (or a linear combination of them) at either boundary. Nevertheless, suppose we choose the Neumann condition. At $x=\tau=0$ we have

$$
\begin{aligned}
\frac{\partial \beta}{\partial x}(0,0) & =-\frac{1}{\sqrt{2}} \frac{\exp \left(z_{0} / \sqrt{2}\right)}{\left(1+\exp \left(z_{0} / \sqrt{2}\right)\right)^{2}} \\
& \approx-\frac{1}{\sqrt{2}} \exp \left(-z_{0} / \sqrt{2}\right)
\end{aligned}
$$

where the last expression holds when $z_{0} \gg 0$. Hence $\beta^{\prime}(0,0)$ can be made arbitrarily small by choosing $z_{0}$ large enough. By choosing the wave to start far enough away from the left boundary we can enforce the Neumann condition to a certain degree of accuracy there, e.g., $\left|\beta^{\prime}(0,0)\right| \approx 10^{-3}$ gives $z_{0} \approx 9.3$.

Similarly, we choose the time of the simulation such that the wave stops before it comes close to the right boundary. Hence we choose

$$
z_{0}=10, \quad x_{b}=30, \quad T=\sqrt{2}\left(x_{b}-2 z_{0}\right)=14.1 .
$$

In addition we set $D=1, k=2.5 \times 10^{-5}$, and $a_{0}=200$, giving a total population of 6000 molecules. The wave starts off at $x=10$ at $t=0$ and travels to $x=20$ at $t=14.1$. The distribution of molecules for a typical run at time $T=14.1$ for $E=60$ is shown in Figure 9. Note the large amount of variation in the data. The average solution over 1000 runs is shown versus the exact solution (52) also in Figure 9. The reaction count in these simulations is about $3.3 \times 10^{5}$ diffusion events for both $\mathrm{A}$ and $\mathrm{B}$ and about $2 \times 10^{3}$ occurrences of the autocatalytic reaction. Each simulation required about $1.5 \mathrm{sec}-$ onds of CPU time. We can estimate the inequality (6) on this mesh by taking the values of $A$ and $B$ at the center of the wavefront, where the majority of the reactions take place. At this point the populations are roughly 50 molecules per element so that the left hand side is $h^{2} / 2 D=1 / 8$ while the right hand side is approximately $1 /\left(k A^{2} B h^{-2}\right) \approx 1.3$.

To test the sampling and spatial discretization error we use the same domain, set $a_{0}=100, k=10^{-4}, D=1$, and

$$
z_{0}=14, \quad x_{b}=30, T=\sqrt{2}\left(x_{b}-2 z_{0}\right)=2.83 .
$$

The wave travels a distance of only $x=2$, from one side of the center of the grid to the other. This gives a smaller value of $\beta^{\prime}$ at the boundary, about $5 \times 10^{-5}$, which insures that this error will be smaller than the one which is being measured. The error measures (33) are shown in Figure 10 on a 40 element evenly spaced mesh for $M \leq 5 \times 10^{5}$. The error measures are taken with respect to the concentration of $\mathrm{B}$ over the interval $[0,18.5]$ rather than the entire domain in order to avoid the region of very small concentration at the right end. The convergence of the spatial error in this case is not as clear as in the pure diffusion case but it appears to be at a somewhat higher rate, although this could be an artifact of the small meshes we are restricted to due to the slow convergence of the sampling error.

\section{B. Dilute Reaction Times}

The previous examples were computed on meshes for which the number of molecules per element was fairly large. In this situation all of the elements along the wavefront will contain enough reactant molecules to execute the autocatalytic reaction. One possible concern is that on very dense meshes there will be few elements which satisfy this criterion and the more complex reactions in a system will never be executed. In this final example we show that this is unlikely to be a problem. Consider the autocatalytic system on the unit interval and let the initial population consist of one molecule of A and two of B. The molecules are randomly placed in elements with equal probability (i.e., the algorithm of Section V A is used where the concentration functions are constant). The molecules are allowed to randomly walk over the mesh until all three of them occupy the same element 

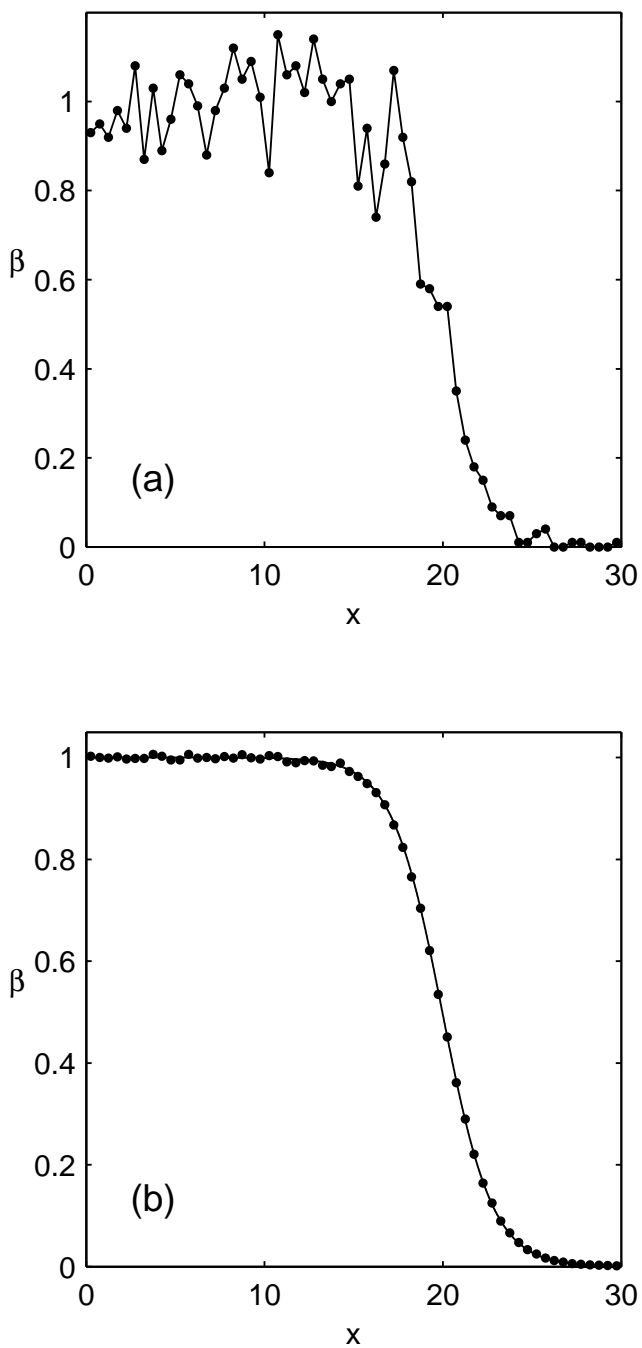

FIG. 9: A typical distribution of molecules at time $T=14.1$ is shown in (a) on a 60 element evenly space mesh. The average distribution over 1000 runs is shown in (b) (solid circles) along with the exact solution (solid line).

and the algorithm determines that the autocatalytic reaction is executed. Using (2) and (6) the average time until the reaction occurs is $\tau=(2 k)^{-1}$. In Table I we show $\tau$ when $k=1 / 2$ on a series of meshes with $E$ ranging from 1 to 100 . The diffusion coefficient has been set to $D=10$ so that the average diffusion time across the mesh is much smaller than the expected reaction time. The results are averaged over 1000 runs.

\section{CONCLUSION}

We have examined an extension of the Gillespie algorithm for simulating reaction-diffusion systems on irregu-
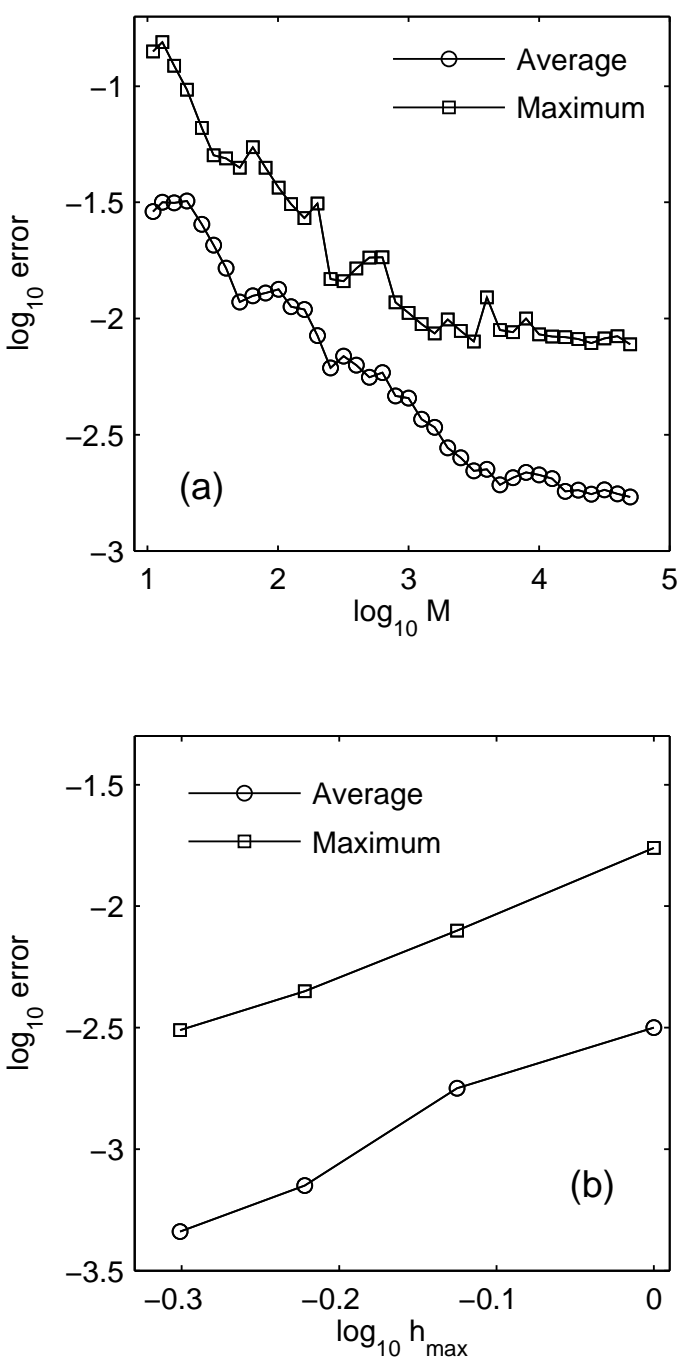

FIG. 10: Convergence of the error for the cubic autocalytic reaction. In (a) we show the error measures (33) on a 40 element evenly spaced mesh for $M \leq 50000$. In (b) the asymptotic value of $\bar{e}_{M}$ and $\hat{e}_{M}$ are plotted versus the element size for $30 \leq E \leq 60$. Fits to the data have slope 2.9 and 2.5 respectively.

\begin{tabular}{|c|ccccccc|}
\hline$E$ & 1 & 2 & 5 & 10 & 25 & 50 & 100 \\
\hline$\tau$ & 1.06 & 1.07 & 1.07 & 1.04 & 1.03 & 1.09 & 0.99 \\
\hline
\end{tabular}

TABLE I: Average time for a single execution of the autocatalytic reaction on a series of evenly spaced meshes of varying size. The expected time is $\tau=1$.

larly spaced Cartesian meshes. The systems may have inhomogeneous diffusion coefficients, including those with discontinuities. We have shown numerical examples in one dimension which demonstrate that the algorithm is capable of simulating both pure diffusion and the nonlinear cubic autocatalytic reaction-diffusion system ac- 
curately in an appropriate limit. We have also shown that the algorithm reproduces the statistics of Brownian motion for the random walk of a single molecule, at least on a sufficiently fine grid. We show that the spatial error caused by the discretization of the domain scales as $h^{2}$ for the diffusion problem and at least this fast for the cubic autocatalytic reaction. It should be stressed that we have not derived any general formulae for the scaling of this error for an arbitrary reaction-diffusion system. Currently this must be determined empirically and the method used in this paper for determining this rate is not guaranteed to work in a general setting.

Although the examples shown in this paper are in one space dimension the method itself trivially generalizes to higher dimensional Cartesian meshes. However, in a general situation such as an irregularly shaped cell or a microchamber of complex geometry one would like to use an unstructured grid. The derivation of the diffusive rate constants by the finite volume method opens up the possibility that one can borrow more results from the finite volume literature to generalize the method to unstructured meshes. Possibilities along these lines include Voronoi cells [27, 28] and irregular polygons based on underlying simplicial meshes $[29,30]$. Such meshes have many advantages over regular Cartesian meshes including the fact that they can be graded in element size, locally refined and unrefined, and that they approximate the domain boundaries much more accurately.

In addition, the use of automatic mesh refinement would seem to be very useful in future versions of the algorithm. For instance, in the simulations of the autocatalytic reaction the variation in the concentrations of $\mathrm{A}$ and $\mathrm{B}$ are greatest in the neighborhood of the wavefront which would seem to be a reasonable place to concentrate the elements. Elsewhere the ratio of A to B is either very large or very small so the number of reactions is small and in these regions it would be preferable to have fewer elements. In addition, in three dimensions the scaling of the diffusion rate is such that a large element will experience fewer diffusion events per unit time than a large number of smaller elements with the same total volume and population. Controlling the element size to take advantage of this may result in a substantial overall saving in simulation time.

There are other reasons for controlling the element size as well. For instance, up to this point we have ignored the finite size of the reactants. If an element becomes too small, it may become "overcrowded" and violate the basic assumption that the reactants collide much more frequently with the solvent than they do with each other. An extreme case would be an element too small to contain even a single reactant molecule. And of course elements cannot be too large either since the inequality (7) will be violated.

We must also be aware, however, that these restrictions on the element size may in some cases be mutually exclusive. This may happen for instance in systems characterized by a large separation of time scales, i.e., rate constants and diffusion coefficients which differ by many orders of magnitude. To be fair, these conditions arise in a wide variety of physical systems and their accurate simulation is an active area of work in many fields.

To summarize, this work can be considered an early step in the simulation of reaction-diffusion systems using the Gillespie algorithm. Many important issues remain to be resolved such as the extension to unstructured meshes, the effectiveness of automatic mesh refinement, and the addition of more realistic features to the basic model such thermodynamics and time varying domain geometry.

\section{Acknowledgements}

Thanks to Mayya Tokman for many helpful discussions and to Naida Lacevic for a useful conversation.
[1] D.T. Gillespie, A General Method for Numerically Simulating the Stochastic Time Evolution of Coupled Chemical Reactions, J. Comp. Phys., 22 (1976), pp. 403-434.

[2] D.T. Gillespie, Exact Stochastic Simulation of Coupled Chemical Reactions, J. Phys. Chem., 81 (1977), pp. 23402361.

[3] J.A. Pelesko and D. Bernstein Modeling MEMS and NEMS (2002), CRC Press, Baton Rouge.

[4] Q. Ouyang, R. Li, G. Li, and H.L. Swinnery, Dependence of Turing Pattern Wavelength on Diffusion Rate, J. Chem. Phys., 102 (1995), pp. 2551-2555.

[5] J. Elf, A. Doncic, M. Ehrenberg Mesoscopic reactiondiffusion in intracellular signaling, SPIE First Int. Symp. Fluctuations and Noise (2003), pp. 114-124.

[6] T. Fricke and J. Schnakenberg, Monte-Carlo simulation of an inhomogeneous reaction-diffusion system in the biophysics of receptor cells, Z. Phys. B., 83 (1991), pp. 277284.
[7] J.J. Lukkien, J.P.L. Segers, P.A.J. Hilbers, R.J. Gelten, and A.P.J. Jansen, Efficient Monte Carlo Methods for the Simulation of Catalytic Surface Reactions, Phys. Rev. E., 58 (1998), pp. 2598-2610.

[8] F. Baras and M.M. Mansour, Reaction-Diffusion Master Equation: A Comparison with Microscopic Simulations, Phys. Rev. E., 54 (1996), pp. 6139-6148.

[9] J. Weimar Three-dimensional Cellular Automata for Reaction-Diffusion Systems, Fundam. Inform., 52 (2002), pp. $277-284$.

[10] G. Turk Generating Textures on Arbitrary Surfaces Using Reaction-Diffusion, Comp. Graphics, 25 (1991), pp. 289298. (SIGGRAPH' '91)

[11] D.T. Gillespie, A Rigorous Derivation of the Chemical Master Equation, Physica A., 188 (1992), pp. 404-425.

[12] B. Cockburn and P.A. Gremaud A Priori Error Estimates for Numerical Methods for Scalar Conservations Laws, Math. Comp. 66 (1997), pp. 547-572. 
[13] S. Rajasekaran and K.W. Ross, Fast Algorithms for Generating Discrete Random Variates with Changing Distributions ACM Transactions on Modeling and Computer Simulations, 3, 1, pp. 1-19 (1993).

[14] M.A. Gibson and J. Bruck, Efficient Exact Stochastic Simulation of Chemical Systems with Many Species and Many Channels, J. Phys. Chem. A. 104 (2000), pp. 18761889.

[15] D. Givon, R. Kupferman, A.M. Stuart, Extracting Macroscopic Dynamics: Model Problems and Algorithms, preprint, 2004.

[16] C. Haselwandter and D. Vvedensky, Fluctuations in the lattice gas for Burgers' equation, J. Phys. A., 35 (2002), pp. L579-L584.

[17] D. Vvedensky, Edwards-Wilkinson equation from lattice transition rules, Phys. Rev. E., 67 (2003), 025102(R).

[18] P.A. Maksym, Fast Monte Carlo Simulation of MBE Growth, Semicond. Sci. Technol., 3 (1988), 594-596.

[19] K.A. Fichthorn and W.H. Weinberg, Theoretical Foundations of Dynamical Monte Carlo Simulations, J. Chem. Phys., 95 (1991), 1090-1096.

[20] J.L. Blue, I. Beichl, F. Sullivan, Faster Monte Carlo Simulations, Phys. Rev., E51 (1995) R867-868.

[21] P. Gray and S.K. Scott, Chemical Oscillations and Instabilities (1990), Clarendon Press, Oxford.

[22] W.H. Press, S.A. Teukolsky, W.T. Vetterling, and B.P. Flannery, Numerical Recipes in C (1993), Cambridge
University Press, Cambridge.

[23] P. L'Ecuyer, Tables of Maximally-Equidistributed Combined LFSR Generators, Math. Comp., 68 (1999), pp. 261-269.

[24] I. Prigogine and R. Lefever, Symmetry Breaking Instabilities in Dissipative Systems. II, J. Chem. Phys., 48 (1968), $1695-1700$

[25] G. Nicolis and I. Prigogine, Self-organization in Nonequilibrium Systems (1977), Wiley, New York.

[26] Z. Cai, J. Douglas, M. Park Development and Analysis of Higher Order Finite Volume Methods Over Rectangles for Elliptic Equations, Advances Comp. Math., 19 (2003), 333.

[27] I.D. Mishev Finite Volume Methods on Voronoi Meshes, Numer. Meth. Part. Diff. Equations, (1998), 193-212.

[28] N. Sukumar, Voronoi Cell Finite Difference Method for the Diffusion Operator on Arbitrary Unstructured Grids, Int. J. Num. Meth. Eng., 57 (2003), 1-34.

[29] I. Aavatsmark, T. Barkve, O. Boe, and T. Mannseth Discretization on Unstructured Grids for Homogeneous, Anisotropic Media, SIAM J. Sci. Comput., 19 (1998), 1700-1716

[30] R.D. Lazarov and S.Z. Tomov, Adaptive Finite Volume Element Method for Convection-Diffusion-Reaction Problems in 3-D, preprint. 